\title{
Validação de um método analítico para a determinação de substâncias ativas em formulações farmacêuticas empregadas em "peelings" químicos
}

\author{
Tatiane Rodrigues Ramos, Maria Inês Rocha Miritello Santoro*, \\ Erika Rosa Maria Kedor-Hackmann, Anil Kumar Singh
}

Departamento de Farmácia, Faculdade de Ciências Farmacêuticas, Universidade de São Paulo

*Correspondência:

M. I. R. M. Santoro

Departamento de Farmácia, Faculdade

de Ciências Farmacêuticas,

Universidade de São Paulo

Caixa Postal 66355,

05389-970, SP, São Paulo, Brasil

E-mail: ines@usp.br
Nos "peelings" químicos utilizam-se formulações esfoliantes, empregadas na terapêutica de queratoses actínicas, rugas, discromias pigmentares, acne vulgar e rosácea. Na presente pesquisa, foram empregadas como amostras, a solução de Jessner (SJ) composta por resorcinol (RS) 14\%, ácido salicílico (AS) $14 \%$ e ácido láctico (AL) $14 \%$ em solução alcoólica e géis de AS a 20\% e RS a 30\%. As técnicas utilizadas foram a espectrofotometria derivada no UV de primeira e segunda ordens em etanol absoluto para o $A S$ e $R S$, respectivamente na SJ, e a espectrofotometria derivada no UV de primeira ordem em ácido sulfúrico 0,1 N para o AS e RS nos géis. Para o AS na SJ, o coeficiente de correlação (r) foi de 0,9999, a precisão expressa pela média dos desvios padrão relativos (DPR) de 0,68\% e a exatidão expressa pela recuperação média de 100,5\%. Para o RS na SJ o r foi de 0,9999, a média dos DPR de 0,83\% e a recuperação média de 100,3\%. No gel de AS, o $r$ foi de 0,9999, a média dos DPR de 0,28 e a recuperação média de 99,3\%. No gel de RS, o r foi de 0,9998, a média dos DPR de 0,34 e a recuperação média de $99,9 \%$.
Uniterms

- "Peelings" químicos

- Ácido salicílico

- Resorcinol

- Espectrofotometria derivada

\section{INTRODUÇÃO}

Ampla gama de substâncias químicas, como os alfahidroxiácidos, beta-hidroxiácidos, derivados fenólicos e retinóides, é utilizada em formulações para "peelings" químicos. O ácido salicílico, resorcinol e ácido láctico (Figuras $1 \mathrm{a}, 1 \mathrm{~b}$ e $1 \mathrm{c}$, respectivamente) são princípios ativos empregados em formulações esfoliantes para a execução de "peelings" químicos e que têm como objetivo produzir uma lesão controlada na pele. Estas substâncias são utilizadas no tratamento das queratoses e rugas actínicas, discromias pigmentares, acnes vulgar e rosácea (Katsambas, Stratigos, 2001; Monheit, 2001). Por provocarem diferentes reações adversas e constituírem diversas associações de princípios ativos, faz-se necessário o desenvolvimento e validação de métodos analíticos para determinação dessas substâncias.

A Solução de Jessner (SJ) é uma formulação clássica de aplicação magistral (Batistuzzo et al., 2000) e os géis de ácido salicílico e de resorcinol a $20 \%$ e 30\%, respectivamente, também são de uso comum na terapêutica (Brody, 2000). Poucos métodos têm sido desenvolvidos 
para determinações de ácido salicílico e de resorcinol associados em formulações farmacêuticas empregadas para "peelings". Técnicas eletroforéticas (Gomez et al., 2003) e cromatográficas (Gou, Zhou, 1998), já foram empregadas para determinar as referidas substâncias em outras matrizes.

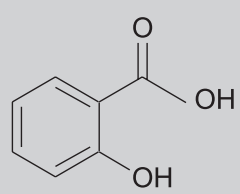

(a)

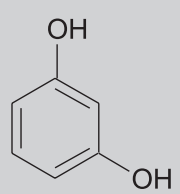

(b)

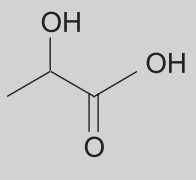

(c)
FIGURA 1 - Estruturas químicas do ácido salicílico (a), resorcinol (b) e ácido láctico (c) .

\section{MATERIAL E MÉTODOS}

\section{Reagentes e amostras}

Ácido salicílico, resorcinol e ácido láctico com pureza de $99 \%, 100,5 \%$ e $88 \%$, respectivamente, etanol absoluto (Labsynth ${ }^{\circledR}$, São Paulo/Brasil), ácido sulfúrico concentrado 96-98\% grau analítico (Merck ${ }^{\circledR}$, Darmstadt, Alemanha) solução de ácido sulfúrico $0,1 \mathrm{~N}$. Solução de Jessner composta por resorcinol (RS) 14\%, ácido salicílico (AS) 14\% e ácido láctico (AL) 14\% em solução alcoólica (amostras A e B, preparada em laboratório e comercial, respectivamente), gel de ácido salicílico 20\% (amostras C e D, preparadas em laboratório e comercial, respectivamente) e gel de resorcinol 30\% (amostras E e F, preparada em laboratório e comercial, respectivamente) foram as amostras selecionadas para a pesquisa.

\section{Equipamento e condições analíticas}

Utilizou-se espectrofotômetro UV-Vis (Shimadzu ${ }^{\circledR}$ UV-160), células de quartzo de $1,0 \mathrm{~cm}$ de caminho óptico. Usaram-se sinais na derivada de primeira ordem em $320,0 \mathrm{~nm}$ e sinais na segunda derivada a $280,0 \mathrm{~nm}$ para o ácido salicílico e resorcinol em SJ, respectivamente. Sinais a $317,2 \mathrm{~nm}$ e $257,6 \mathrm{~nm}$ na primeira derivada foram utilizados para o ácido salicílico e resorcinol em gel, respectivamente.

\section{Preparo das soluções padrão}

Para a solução de Jessner, a curva padrão do ácido salicílico foi obtida pesando-se exatamente cerca de $100,0 \mathrm{mg}$ que foram transferidos para balão volumétrico de $50 \mathrm{~mL}$ e o volume completado com etanol absoluto. Transferiramse 5,0 $\mathrm{mL}$ para balão volumétrico de $50 \mathrm{~mL}$ completandose o volume com o mesmo solvente. Desta última solução, transferiram-se 7 alíquotas diferentes para balões volumétricos de $100 \mathrm{~mL}$ completando-os com etanol absoluto. Foram obtidas soluções com intervalo de concentração entre 12,0 e 24,0 $\mu \mathrm{g}$ de ácido salicílico/mL. A curva padrão do resorcinol realizou-se pesando exatamente cerca de $100,0 \mathrm{mg}$, que foram transferidos para balão volumétrico de $50 \mathrm{~mL}$. Obtiveram-se soluções com intervalo de concentração entre 22,0 e 34,0 $\mu$ g de resorcinol $/ \mathrm{mL}$, através do mesmo esquema de diluição descrito.

Para o gel de ácido salicílico, realizou-se a curva padrão pesando-se exatamente cerca de 100,0 mg de ácido salicílico, que foram transferidos para balão volumétrico de $100 \mathrm{~mL}$. O volume foi completado com $\mathrm{H}_{2} \mathrm{SO}_{4} 0,1 \mathrm{~N}$. Transferiram-se $10,0 \mathrm{~mL}$ para balão volumétrico de $100 \mathrm{~mL}$ e completou-se com $\mathrm{H}_{2} \mathrm{SO}_{4}$ 0,1 $\mathrm{N}$. Desta última solução, transferiram-se 7 alíquotas diferentes para balões volumétricos de $25 \mathrm{~mL}$ completando-os com o mesmo solvente. Foram obtidas soluções com intervalo de concentração entre 8,0 e 32,0 $\mu \mathrm{g}$ de ácido salicílico $/ \mathrm{mL}$.

Para o gel de resorcinol, realizou-se a curva padrão pesando exatamente cerca de 100,0 mg de resorcinol, que foram transferidos para balão volumétrico de $100 \mathrm{~mL}$, levando-se à marca com $\mathrm{H}_{2} \mathrm{SO}_{4} 0,1 \mathrm{~N}$. Transferiram-se 25,0 mL para balão volumétrico de $250 \mathrm{~mL}$ e completou-se com $\mathrm{H}_{2} \mathrm{SO}_{4} 0,1 \mathrm{~N}$. Desta última solução, transferiram-se 7 alíquotas diferentes para balões volumétricos de $50 \mathrm{~mL}$ completando-os com o mesmo solvente, obtendo-se soluções com intervalo de concentração entre 18,0 e 42,0 $\mu \mathrm{g} / \mathrm{mL}$ de resorcinol.

\section{Preparo das soluções das amostras}

Para a determinação do ácido salicílico em SJ transferiram-se 4,0 mL de amostra para balão volumétrico de $100 \mathrm{~mL}$. Completou-se o volume com etanol absoluto. Transferiu-se uma alíquota de $4,0 \mathrm{~mL}$ para balão volumétrico de $50 \mathrm{~mL}$ e desta última solução transferiu-se uma alíquota de 4,0 mL para balão volumétrico de $100 \mathrm{~mL}(17,92 \mu \mathrm{g}$ de ácido salicílico/mL). Para a determinação do resorcinol, transferiram-se 5,0 mL de SJ para balão volumétrico de $100 \mathrm{~mL}$ e adicionou-se etanol absoluto até a marca. Transferiu-se uma alíquota de 5,0 $\mathrm{mL}$ para balão volumétrico de $100 \mathrm{~mL}$ e desta ultima solução transferiu-se uma alíquota de 4,0 $\mathrm{mL}$ para balão volumétrico de $50 \mathrm{~mL}(28,0 \mu \mathrm{g}$ de resorcinol $/ \mathrm{mL}$ ).

Para o gel de ácido salicílico, foi pesado, do gel, o equivalente a 20,0 mg de ácido salicílico e transferiu-se para balão volumétrico de $100 \mathrm{~mL}$. O volume foi comple- 
tado com $\mathrm{H}_{2} \mathrm{SO}_{4} 0,1 \mathrm{~N}$. Transferiram-se alíquotas de $5,0 \mathrm{~mL}$ para balões volumétricos de $50 \mathrm{~mL}(20,0 \mu \mathrm{g}$ de ácido salicílico $/ \mathrm{mL}$ ).

Para o gel de resorcinol, foi pesado, do gel, o equivalente a 30,0 mg de resorcinol e transferiu-se para balão volumétrico de $100 \mathrm{~mL}$. O volume foi completado com $\mathrm{H}_{2} \mathrm{SO}_{4} 0,1 \mathrm{~N}$. Transferiram-se alíquotas de 5,0 $\mathrm{mL}$ para balões volumétricos de $50 \mathrm{~mL}(30,0 \mu \mathrm{g}$ de resorcinol $/ \mathrm{mL})$.

\section{Teste de recuperação (preparo das soluções)}

$\mathrm{Na}$ SJ foram preparadas, separadamente, soluções padrão e soluções amostra com 70,0 e 56,0 $\mu \mathrm{g} / \mathrm{mL}$ de ácido salicílico e resorcinol, respectivamente. Alíquotas fixas de soluções da amostra e variáveis de soluções padrão foram transferidas para balões volumétricos de 50 e $25 \mathrm{~mL}$ na determinação do ácido salicílico e resorcinol, respectivamente. Nas amostras gel, prepararam-se separadamente soluções padrão e soluções da amostra de 70,0 e 90,0 $\mu \mathrm{g} /$ $\mathrm{mL}$ de ácido salicílico e resorcinol, respectivamente. Alíquotas fixas de soluções da amostra e variáveis de soluções padrão foram transferidas para balões volumétricos de $50 \mathrm{~mL}$ para posterior determinação.

\section{Validação dos métodos}

Os métodos foram validados segundo as diretrizes da "International Conference on Harmonization (ICH)" (ICH, 2001) e AOAC International (AOAC, 2000).

\section{Linearidade}

Foi determinada pelas curvas padrão, as quais foram obtidas em 7 níveis de concentração $(12,0-24,0 \mu \mathrm{g} / \mathrm{mL}$ e $22,0-34,0 \mu \mathrm{g} / \mathrm{mL}$ para o ácido salicílico e resorcinol em SJ, respectivamente; $8,0-32,0 \mu \mathrm{g} / \mathrm{mL}$ para o ácido salicílico em gel e $18,0-42,0 \mu \mathrm{g} / \mathrm{mL}$ para o resorcinol em gel). As soluções foram preparadas em triplicata e a linearidade foi avaliada pelo método dos mínimos quadrados.

\section{Precisão}

Foi determinada pela repetibilidade, utilizando todas as amostras nas mesmas concentrações, no mesmo dia. Foram preparadas e analisadas dez soluções de cada amostra e utilizado o desvio padrão médio como parâmetro estatístico.

\section{Exatidão}

Determinada pelo teste de recuperação. Todas as soluções foram preparadas em triplicata e a porcentagem de recuperação foi calculada empregando-se a equação proposta pela AOAC International (AOAC, 2000).

\section{Especificidade}

Determinada para todos os métodos. Foram utilizados placebos (Jenke, 1996) (etanol absoluto para SJ e gel base para o gel de ácido salicílico e resorcinol). Avaliaramse os sistemas a fim de observar respostas instrumentais ou sobreposição de bandas nos espectros.

\section{Limite de detecção e limite de quantificação}

Os limites de detecção (LD) e de quantificação (LQ) foram determinados com base no desvio padrão da resposta instrumental (desvio padrão do residual da linha de regressão) e sua relação com a inclinação da reta na curva padrão construída a baixas concentrações (Swartz, Krull, 1998).

\section{RESULTADOS E DISCUSSÃO}

Os métodos validados são simples, rápidos e de baixo custo. Na quantificação do ácido salicílico na SJ, observou-se que na derivada de primeira ordem existe um mínimo a 320,0 nm, que corresponde unicamente a esta substância. Etanol absoluto, resorcinol e ácido láctico não apresentaram resposta instrumental nesta região nas condições descritas (Figura 2).

Para o ácido salicílico, os dados obtidos para a linearidade, pelo método dos mínimos quadrados, demonstraram um bom coeficiente de correlação (Tabela I). Os valores experimentais obtidos na quantificação indicam variabilidade satisfatória (repetibilidade) (Tabela II). Uma boa exatidão do método foi comprovada pelos testes de recuperação (Tabela III). O método mostrou-se sensível para pequenas quantidades de ácido salicílico presentes na SJ (Tabela I) e não foi observada interferência das outras substâncias que constituem a SJ.

$\mathrm{Na}$ determinação quantitativa do resorcinol em SJ pela derivada de segunda ordem, observou-se um máximo a 280,0 nm, que corresponde unicamente à referida substância. Nesta região, o etanol absoluto, o ácido salicílico e o ácido láctico não apresentam resposta instrumental, não interferindo, portanto, na determinação (Figura 3).

Para o resorcinol, os dados obtidos pelo método dos mínimos quadrados demonstram um bom coeficiente de correlação (Tabela I). Os valores obtidos na quantificação indicam satisfatória repetibilidade (Tabela II). Uma boa exatidão do método foi comprovada pelos testes de recuperação (Tabela III). O método mostrou-se sensível a pe- 


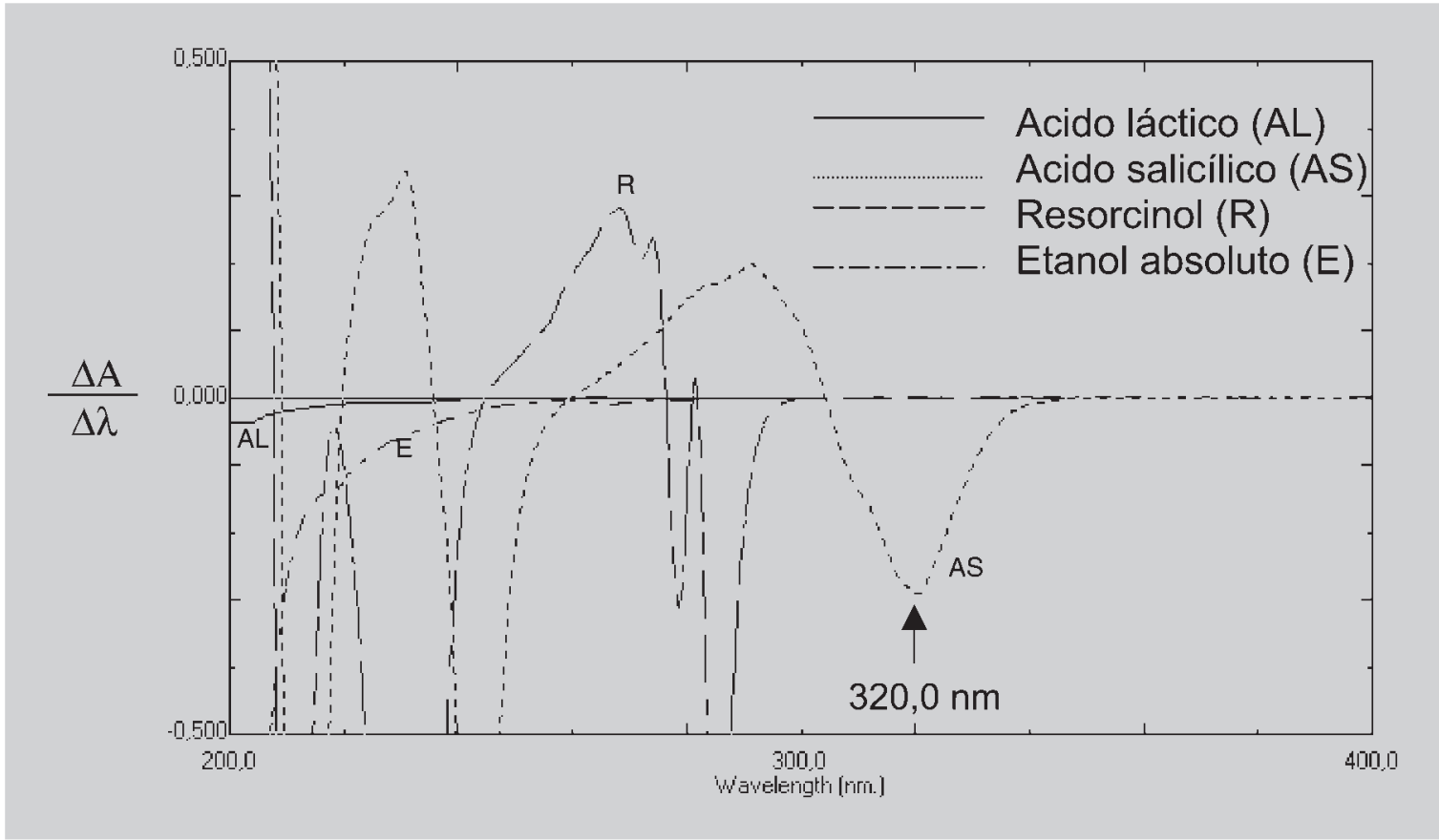

FIGURA 2 - Espectros no UV na primeira derivada do ácido salicílico $(18,0 \mu \mathrm{g} / \mathrm{mL})$, resorcinol $(28,0 \mu \mathrm{g} / \mathrm{mL})$ e ácido láctico $(18,0 \mu \mathrm{g} / \mathrm{mL})$ em etanol absoluto.

TABELA I - Resultados estatísticos obtidos na validação do método para a determinação do ácido salicílico e do resorcinol em SJ.

\begin{tabular}{lcc}
\hline Parâmetro estatístico & Acido salicílico $^{\mathbf{a}}$ & Resorcinol $^{\mathbf{b}}$ \\
\hline Faixa de concentração $(\mu \mathrm{g} / \mathrm{mL})$ & $12,0-24,0$ & $22,0-34,0$ \\
Equação da reta & $\mathrm{Y}=0,0165 \mathrm{x}-0,00595$ & $\mathrm{Y}=0,0071 \mathrm{x}-0,00346$ \\
Coeficiente de correlação $(\mathrm{r})$ & 0,9999 & 0,9999 \\
Limite de detecção $(\mu \mathrm{g} / \mathrm{mL})$ & 0,19 & 0,27 \\
Limite de quantificação $(\mu \mathrm{g} / \mathrm{mL})$ & 0,65 & 0,91 \\
\hline
\end{tabular}

${ }^{\mathrm{a}}$ Determinações na primeira derivada; ${ }^{\mathrm{b}}$ Determinações na segunda derivada

TABELA II - Resultados obtidos na determinação quantitativa do ácido salicílico e do resorcinol na SJ.

\begin{tabular}{lcccc}
\hline Solução de Jessner & \multicolumn{2}{c}{ Acido salicílico $^{\text {a }}$} & \multicolumn{2}{c}{ Resorcinol $^{\mathbf{a}}$} \\
& $(\mathbf{g} / \mathbf{1 0 0} \mathbf{m L})$ & $\mathbf{D P R}^{\mathbf{b}} \mathbf{\%}$ & $\mathbf{( g / 1 0 0 m L )}^{\mathbf{D P R}^{\mathbf{b}} \mathbf{~ \% ~}}$ \\
\hline Amostra A & 14,57 & 0,67 & 14,75 & 0,84 \\
Amostra B & 14,31 & 0,70 & 14,48 & 0,81 \\
\hline
\end{tabular}

${ }^{a}$ Média de dez determinações; ${ }^{b} \mathrm{DPR}=$ Desvio padrão relativo.

quenas quantidades de resorcinol presentes na SJ (Tabela I) e não foi observada interferência das outras substâncias que constituem a SJ.

No caso do ácido láctico, a espectrofotometria no UV não foi capaz de quantificá-lo na SJ devido às características estruturais. Seria, portanto, necessária a utilização de outro tipo de detecção ou a realização de reação de complexação. De qualquer forma, observou-se que não houve interferência na determinação das outras substâncias em questão (Figuras 2 e 3).

Em relação à quantificação do ácido salicílico e do resorcinol em gel, observou-se que na derivada de primeira ordem existem um mínimo e um máximo a 317,2 nm e $257,6 \mathrm{~nm}$, que correspondem unicamente àquelas substân- 
cias. O gel base não apresentou resposta instrumental nestes comprimentos de onda empregados nas determinações analíticas (Figura 4).

Para o ácido salicílico e resorcinol em gel, os dados da linearidade pelo método dos mínimos quadrados apresentaram bom coeficiente de correlação (Tabela IV). Os valores experimentais obtidos na quantificação indicam repetibilidade satisfatória (Tabela V). Uma boa exatidão

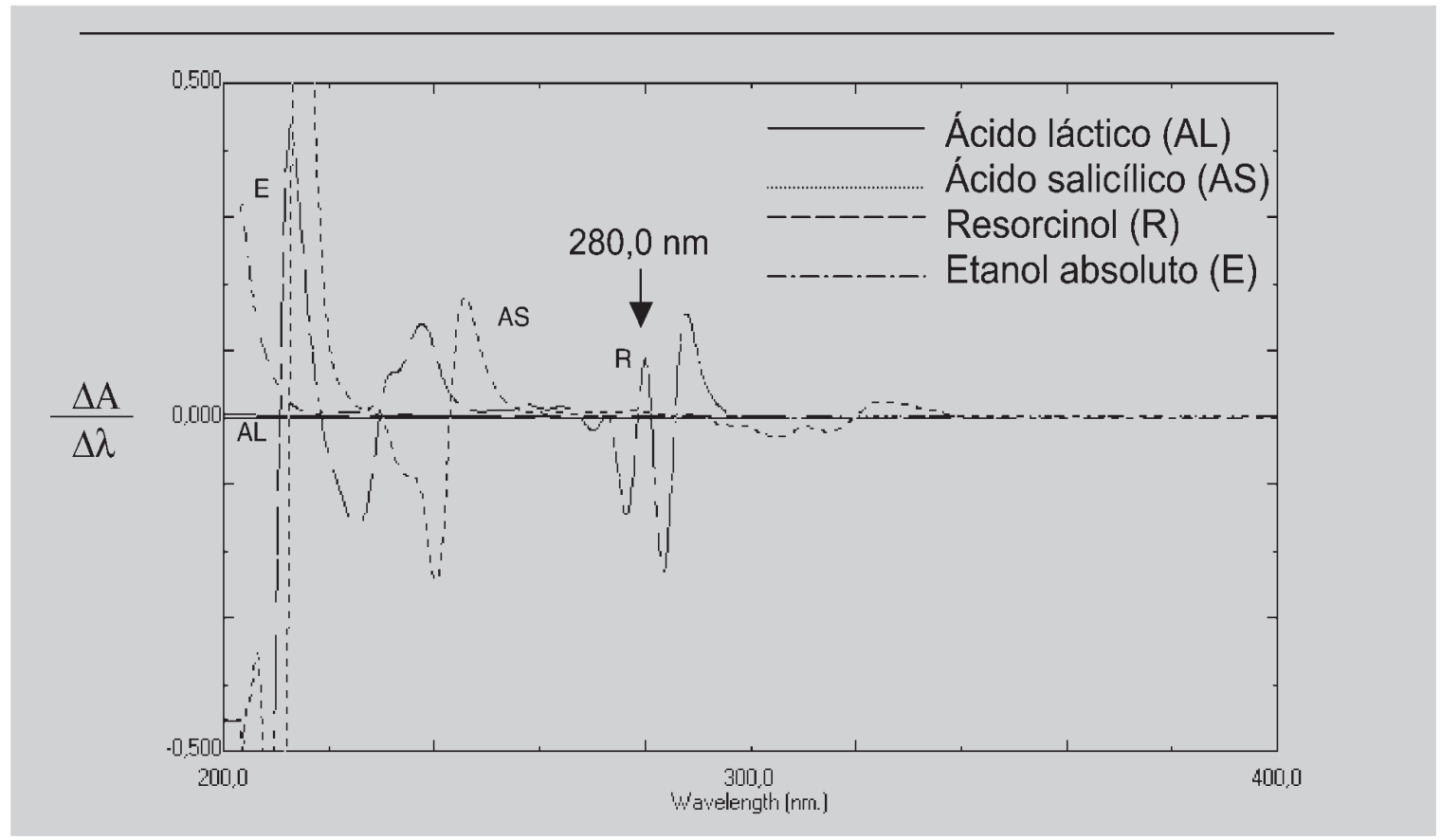

FIGURA 3 - Espectros no UV na segunda derivada do ácido salicílico $(18,0 \mu \mathrm{g} / \mathrm{mL})$, resorcinol $(28,0 \mu \mathrm{g} / \mathrm{mL})$ e ácido láctico $(18,0 \mu \mathrm{g} / \mathrm{mL})$ em etanol absoluto.

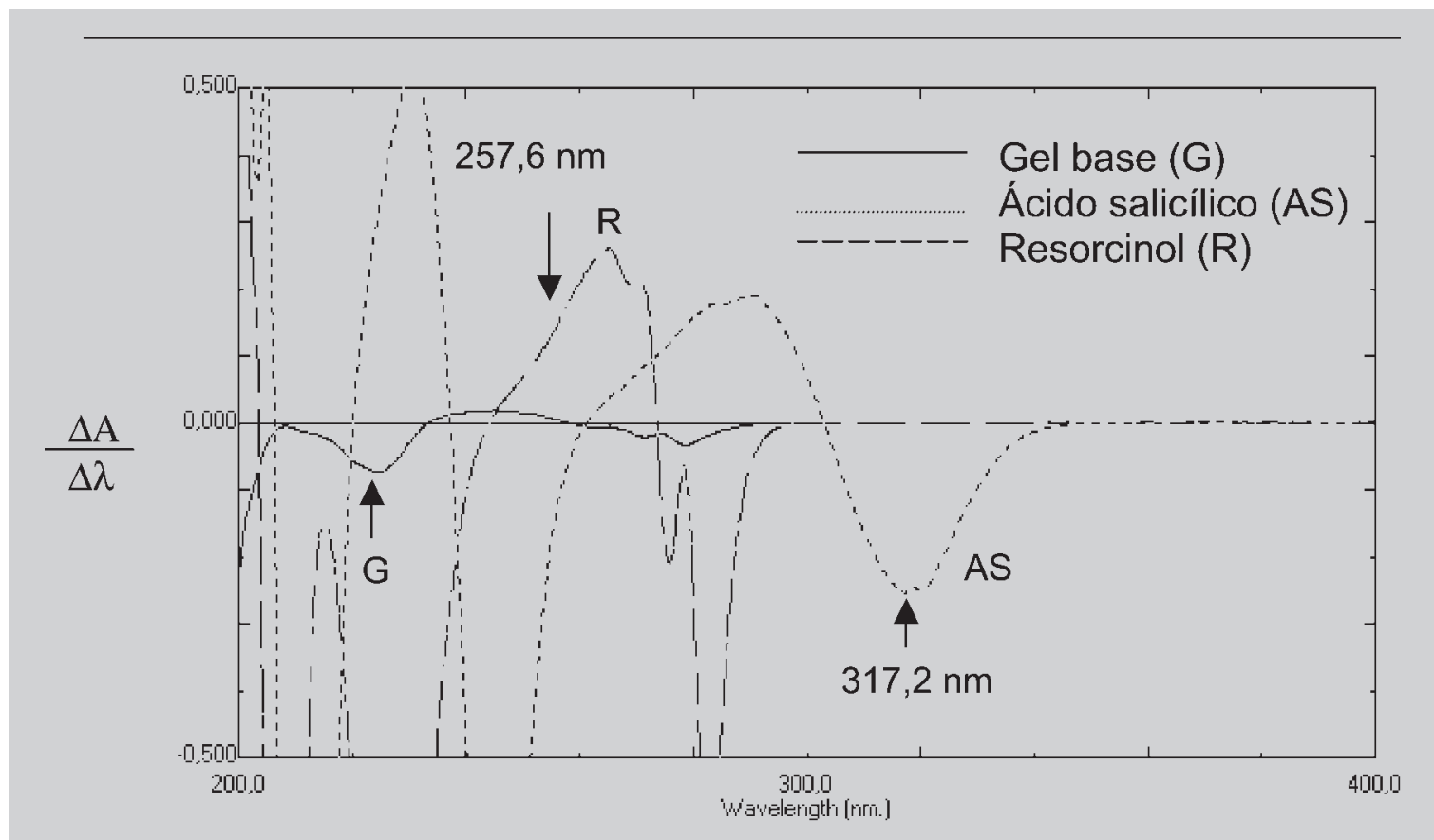

FIGURA 4 - Espectro no UV da primeira derivada do ácido salicílico 20,0 $\mu \mathrm{g} / \mathrm{mL}$ (AS); resorcinol 30,0 $\mu \mathrm{g} / \mathrm{mL}$ (R) e gel base (placebo) $(\mathrm{G}) \mathrm{em}_{2} \mathrm{SO}_{4} 0,1 \mathrm{~N}$. 
TABELA III - Recuperação das soluções padrão de ácido salicílico e de resorcinol adicionada às amostras de SJ e analisadas pelo método proposto

\begin{tabular}{|c|c|c|c|c|c|c|}
\hline \multirow{3}{*}{ Amostra } & \multicolumn{3}{|c|}{ Ácido salicúlico } & \multicolumn{3}{|c|}{ Resorcinol } \\
\hline & \multicolumn{2}{|c|}{ Concentração $(\mu \mathrm{g} / \mathrm{mL})$} & \multirow{2}{*}{$\begin{array}{c}\text { Recuperação }{ }^{\mathrm{a}} \\
(\%)^{\mathrm{a}}\end{array}$} & \multicolumn{2}{|c|}{ Concentração $(\mu \mathrm{g} / \mathrm{mL})$} & \multirow{2}{*}{$\begin{array}{c}\text { Recuperação } \\
(\%)^{\mathrm{a}}\end{array}$} \\
\hline & Adicionada & Recuperada & & Adicionada & Recuperada & \\
\hline \multirow{3}{*}{ A } & 2,0 & 2,06 & $103,0 \pm 0,3$ & 1,0 & 1,01 & $101,0 \pm 0,2$ \\
\hline & 3,0 & 2,98 & $99,3 \pm 0,2$ & 2,0 & 1,98 & $99,0 \pm 0,1$ \\
\hline & 5,0 & 4,99 & $99,8 \pm 0,3$ & 3,0 & 3,04 & $101,3 \pm 0,2$ \\
\hline \multirow{3}{*}{ B } & 2,0 & 2,01 & $100,5 \pm 0,2$ & 1,0 & 0,99 & $99,0 \pm 0,3$ \\
\hline & 3,0 & 3,03 & $101,0 \pm 0,2$ & 2,0 & 2,01 & $100,5 \pm 0,4$ \\
\hline & 5,0 & 4,97 & $99,4 \pm 0,4$ & 3,0 & 3,02 & $100,7 \pm 0,3$ \\
\hline
\end{tabular}

${ }^{a}$ Média de três determinações

TABELA IV - Resultados estatísticos obtidos na validação do método para a determinação do ácido salicílico e do resorcinol em géis

\begin{tabular}{lcc}
\hline Parâmetro estatístico & Acido salicílico $^{\mathbf{a}}$ & Resorcinol $^{\mathbf{b}}$ \\
\hline Faixa de concentração $(\mu \mathrm{g} / \mathrm{mL})$ & $8,0-32,0$ & $18,0-42,0$ \\
Equação da reta & $\mathrm{Y}=0,0128 \mathrm{x}-0,00169$ & $\mathrm{Y}=0,0062 \mathrm{x}-0,00743$ \\
Coeficiente de correlação $(\mathrm{r})$ & 0,9999 & 0,9998 \\
Limite de detecção $(\mu \mathrm{g} / \mathrm{mL})$ & 0,51 & 0,13 \\
Limite de quantificação $(\mu \mathrm{g} / \mathrm{mL})$ & 1,71 & 0,42 \\
\hline
\end{tabular}

TABELA V - Resultados obtidos na determinação quantitativa do ácido salicílico e do resorcinol em géis

\begin{tabular}{lcccc}
\hline Amostra do gel & \multicolumn{2}{c}{ Acido salicílico $^{\text {a }}$} & \multicolumn{2}{c}{ Resorcinol $^{\mathbf{a}}$} \\
& $(\mathbf{g} / \mathbf{1 0 0 g})$ & DPR $^{\mathbf{b}} \mathbf{\%}$ & $\mathbf{( g / 1 0 0 g )}$ & $\mathbf{D P R}^{\mathbf{b}} \mathbf{~ \%}$ \\
\hline Amostra C & 20,36 & 0,26 & & \\
Amostra D & 19,91 & 0,30 & 30,76 & 0,34 \\
Amostra E & & & 30,39 & 0,35 \\
Amostra F & & & \\
\hline
\end{tabular}

${ }^{a}$ Média de dez determinações $\mathrm{DPR}^{\mathrm{b}}=$ Desvio padrão relativo.

TABELA VI - Recuperação das soluções padrão de ácido salicílico e de resorcinol adicionadas às amostras de géis e analisadas pelo método proposto

\begin{tabular}{|c|c|c|c|c|c|c|c|}
\hline \multirow{3}{*}{ Amostra } & \multicolumn{3}{|c|}{ Ácido salicílico } & \multicolumn{4}{|c|}{ Resorcinol } \\
\hline & \multicolumn{2}{|c|}{ Concentração $(\mu \mathrm{g} / \mathrm{mL})$} & \multirow{2}{*}{$\begin{array}{c}\text { Recuperação }{ }^{\mathrm{a}} \\
(\%)^{\mathrm{a}}\end{array}$} & \multirow[t]{2}{*}{ Amostra } & \multicolumn{2}{|c|}{ Concentração $(\mu \mathrm{g} / \mathrm{mL})$} & \multirow{2}{*}{$\begin{array}{c}\text { Recuperação } \\
(\%)^{\mathrm{a}}\end{array}$} \\
\hline & Adicionada & Recuperada & & & Adicionada & Recuperada & \\
\hline \multirow{3}{*}{$\mathrm{C}$} & 4,0 & 3,93 & $98,2 \pm 0,1$ & \multirow{3}{*}{$\mathrm{E}$} & 4,0 & 3,99 & $99,8 \pm 0,2$ \\
\hline & 5,0 & 4,90 & $98,0 \pm 0,3$ & & 5,0 & 5,00 & $100,0 \pm 0,3$ \\
\hline & 10,0 & 10,08 & $100,8 \pm 0,3$ & & 10,0 & 10,00 & $100,0 \pm 0,3$ \\
\hline \multirow{3}{*}{$\mathrm{D}$} & 4,0 & 3,98 & $99,5 \pm 0,2$ & \multirow{3}{*}{$\mathrm{F}$} & 4,0 & 3,93 & $98,2 \pm 0,2$ \\
\hline & 5,0 & 4,97 & $99,4 \pm 0,2$ & & 5,0 & 5,04 & $100,8 \pm 0,2$ \\
\hline & 10,0 & 9,99 & $99,9 \pm 0,3$ & & 10,0 & 10,04 & $100,4 \pm 0,2$ \\
\hline
\end{tabular}

${ }^{a}$ Média de três determinações 
do método foi comprovada nos testes de recuperação (Tabela VI). O método demonstrou ser sensível a pequenas quantidades dos princípios ativos, como indicam os resultados dos limites de detecção e de quantificação (Tabela IV). Não foi observada interferência do placebo dos géis.

\section{CONCLUSÕES}

Os métodos propostos podem ser empregados para a determinação quantitativa do ácido salicílico e do resorcinol em SJ, bem como do ácido salicílico e do resorcinol em géis. Os métodos mostraram-se rápidos, simples, precisos, exatos e sensíveis. Uma vantagem é que estes métodos são de baixo custo e pouco impacto ambiental. Os métodos propostos e validados podem ser utilizados na análise de rotina para o controle de qualidade deste tipo de preparações farmacêuticas.

\section{ABSTRACT \\ Validation of analytical methods for the determination of active substances in pharmaceutical preparations used in chemical peelings}

Chemical peeling is obtained with exfoliating formulations and is used in the treatment of actinic keratosis, wrinkles, dyschromies, acne vulgaris and rosacea acne. In this research we selected the Jessner Solution (JS), a pharmaceutical preparation composed of resorcinol (RS) (14\%), salicylic acid (SA) (14\%) and lactic acid (LA) (14\%) in alcoholic solution and two gel samples composed of RS (30\%) and SA (20\%), respectively. First and second derivative $U V$ spectrophotometric methods were developed and validated for determination of $S A$ and $R S$, respectively in JS alcoholic solution, ethanol was used as background. A first derivative UV spectrophotometric method was developed for determination of these active substances in gel samples using $0.1 \mathrm{~N}$ sulfuric acid as background. For SA in the JS, the correlation coefficient ( $r$ ) was 0.9999, the precision expressed by the relative standard deviation (RSD) of $0.68 \%$ and the accuracy expressed by the average percent recovery of $100.5 \%$. For RS in the JS, the r was 0.9999, the RSD of $0.83 \%$ and average recovery of $100.3 \%$. In the gel of $S A r$ was 0.9999 , the mean RSD was $0.28 \%$ and average recovery of $99.3 \%$. In the gel of $R S$ the $r$ was 0.9998 , mean RSD was $0.34 \%$ and average recovery of $99.9 \%$.
Uniterms: Chemical peelings. Salicylic acid. Resorcinol. Derivative spectrophotometry.

\section{REFERÊNCIAS BIBLIOGRÁFICAS}

ASSOCIATION OF OFFICIAL ANALYTICAL CHEMISTS INTERNATIONAL. Official methods of analysis of the Association of Official Analytical Chemists. 17.ed. Gaithersburg: AOAC, 2000. v.1.

BATISTUZZO, J.A.O.; ITAYA, M.; ETO, Y. Formulário médico-farmacêutico. São Paulo: Tecnopress, 2000 . p.147-229.

BRODY, H.J. "Peeling” químico e "resurfacing". 2.ed. Rio de Janeiro: Reichmann \&Affonso, 2000. p.5,45-51, 120-121.

GOMEZ, R.M.; OLSINA, R. A.; MARTINEZ, L.D.; SILVA, M.F. Simultaneous determination of chloramphenicol, salicylic acid and resorcinol by capillary zone electrophoresis and its application to pharmaceutical dosage forms. Talanta, Amsterdam, v.61, n.2, p.233-238, 2003.

GOU, X.J.; ZHOU, M. Determination of resorcinol and salicylic acid in piyanning tincture by high-performance liquid chromatography. Sepu, Beijing, v.16, n.6, p.532-533, 1998.

ICH Q2b. Text on validation of analytical procedure: methodology. Disponível em: http://www.ich.org. Acesso em: 04 maio 2001.

JENKE, D.R. Chromatographic method validation: a review of current practices and procedures. II. Guidelines for primary validation parameters. J. Liq. Chrom. Rel. Technol., New York, v. 19, n 5, p. 737-757,1996.

KATSAMBAS, A.D.; STRATIGOS, A.J. Dermatologic therapy in the new millennium. Clin. Dermatol., Tokyo, v.19, p.65-67, 2001.

MONHEIT, G.D. Chemical peeling vs. laser resurfacing. Dermatol. Surg., New York, v.27, p.213-214, 2001.

SWARTZ, M.E.; KRULL, I.S. Validação de métodos cromatográficos. Pharm. Technol., São Paulo, v.2, n. 3, p.12-20, 1998.

Recebido para publicação em 17 de maio de 2004. Aceito para publicação em 04 de abril de 2005. 